\title{
Red light imaging for programmed cell death visualization and quantification in plant-pathogen interactions
}

\author{
Sergio Landeo Villanueva ${ }^{1}$ | Michele C. Malvestiti ${ }^{1}$ | Wim van leperen ${ }^{2}$ | \\ Matthieu H. A. J. Joosten (D) ${ }^{1}$ | Jan A. L. van Kan (D) ${ }^{1}$
}

${ }^{1}$ Laboratory of Phytopathology, Wageningen University \& Research, Wageningen, Netherlands

${ }^{2}$ Horticulture and Product Physiology, Wageningen University \& Research, Wageningen, Netherlands

\section{Correspondence}

Jan A. L. van Kan, Wageningen University, Laboratory of Phytopathology, Wageningen University \& Research, Wageningen,

Netherlands.

Email: jan.vankan@wur.nl

Funding information

Consejo Nacional de Ciencia, Tecnología e Innovación Tecnológica, Grant/Award Number: PhD scholarship contract 119-2017-FONDECYT; NWO-ENW, Grant/ Award Number: grant number GSGT. GSGT.2018.008; Fondo Nacional de Desarrollo Científico y Tecnológico

\begin{abstract}
Studies on plant-pathogen interactions often involve monitoring disease symptoms or responses of the host plant to pathogen-derived immunogenic patterns, either visually or by staining the plant tissue. Both these methods have limitations with respect to resolution, reproducibility, and the ability to quantify the results. In this study we show that red light detection by the red fluorescent protein (RFP) channel of a multipurpose fluorescence imaging system that is probably available in many laboratories can be used to visualize plant tissue undergoing cell death. Red light emission is the result of chlorophyll fluorescence on thylakoid membrane disassembly during the development of a programmed cell death process. The activation of programmed cell death can occur during either a hypersensitive response to a biotrophic pathogen or an apoptotic cell death triggered by a necrotrophic pathogen. Quantifying the intensity of the red light signal enables the magnitude of programmed cell death to be evaluated and provides a readout of the plant immune response in a faster, safer, and nondestructive manner when compared to previously developed chemical staining methodologies. This application can be implemented to screen for differences in symptom severity in plant-pathogen interactions, and to visualize and quantify in a more sensitive and objective manner the intensity of the plant response on perception of a given immunological pattern. We illustrate the utility and versatility of the method using diverse immunogenic patterns and pathogens.
\end{abstract}

\section{KEYWORDS}

chlorophyll, hypersensitive response, programmed cell death, RFP, thylakoid disassembly

\section{1 | INTRODUCTION}

In natural environments as well as in agricultural systems, plants are constantly threatened by pathogens, including nematodes, oomycetes, fungi, bacteria, and viruses. In order to invade, colonize, and obtain nutrients from their host, these pathogens must deal with plant defence mechanisms, and therefore they have evolved different lifestyles and strategies to overcome the plant immune system and cause disease. Given their sessile nature, plants rely in the first line of their defence on anatomical barriers and preformed phytochemical compounds to prevent pathogen invasion. However, when pathogens can overcome these first passive defence layers, recognition of the invaders by the plant can take place within the host

Sergio Landeo Villanueva and Michele C. Malvestiti contributed equally to the work.

This is an open access article under the terms of the Creative Commons Attribution-NonCommercial License, which permits use, distribution and reproduction in any medium, provided the original work is properly cited and is not used for commercial purposes.

(c) 2021 The Authors. Molecular Plant Pathology published by British Society for Plant Pathology and John Wiley \& Sons Ltd 
tissue. To perceive danger, plants have evolved a wide range of receptors and their function consists of the recognition of so-called immunogenic patterns (IPs), in both the apoplast and the cytoplasm (Gust et al., 2017; van der Burgh \& Joosten, 2019). Triggers of plant immunity include damage-associated molecular patterns (DAMPs) (Boller \& Felix, 2009), structural components of the invaders (microbe-associated molecular patterns, MAMPs), and pathogen-secreted compounds known as effectors that serve to perturb plant defence responses and promote virulence (Kanyuka \& Rudd, 2019). Apoplastic triggers of immunity are referred to as extracellular immunogenic patterns (ExIPs) (van der Burgh \& Joosten, 2019). These are perceived by trans-membrane pattern recognition receptors (PRRs) located on the cell surface, which have been grouped into receptor-like kinases (RLKs, containing a cytoplasmic kinase domain) and receptor-like proteins (RLPs, which lack such a domain) (Gust et al., 2017). By contrast, cytoplasmic danger signals are referred to as intracellular immunogenic patterns (InIPs) and are recognized by the activity of cytoplasmic receptors, usually from the category of nucleotide-binding leucine-rich repeat receptors (NB-LRRs) (van der Burgh \& Joosten, 2019). Extra- and intracellular receptors possess highly variable LRR domains that confer specific binding capacity to a wide array of ExIPs and InIPs (van der Burgh \& Joosten, 2019). On perception of immunogenic patterns, PRRs and NB-LRRs activate downstream signalling pathways, leading to the induction of immune responses to block the invader.

Plant immune responses comprise multiple cellular processes, among others alkalization of the extracellular space (Granado et al., 1995; Jourdan et al., 2009), cell wall reinforcement via callose deposition (Luna Diez et al., 2010; Voigt, 2014), accumulation of reactive oxygen and nitrogen species (Turkan, 2018), an increase in intracellular calcium (Grant et al., 2000), biosynthesis of pathogenesis-related (PR) proteins (Jain \& Khurana, 2018) and phytoalexins (Jeandet, 2015), and the release of small RNAs to interfere with the transcriptional machinery of the invading pathogen (Cai et al., 2018). In an attempt to stop pathogen invasion and disease development, the affected plant cells can commit suicide by undergoing programmed cell death (PCD). Even though it represents a decisive and seemingly altruistic response of an individual plant cell to pathogen invasion, PCD can lead to either resistance or to disease development, depending on the lifestyle of the pathogen. In incompatible interactions with biotrophic pathogens, PCD is referred to as the hypersensitive response (HR) and manifests itself as an effective resistance response of the plant upon pathogen recognition. The HR represents a form of autophagic PCD, preventing the spread of biotrophic microbes (Kabbage et al., 2017; Michaeli et al., 2016). For example, race 5 of the biotrophic fungus Cladosporium fulvum, which causes leaf mould of tomato following a typical gene-for-gene interaction, cannot cause disease on tomato carrying the gene encoding the RLP Cf-4 (Joosten et al., 1994; Thomas et al., 1997). This PRR recognizes the Avr4 effector protein that is secreted by $C$. fulvum race 5 and mediates the activation of the HR, leading to leaf mould resistance (Thomas et al., 1997). On the other hand, in interactions with necrotrophs, PCD occurs after induction of apoptosis of the affected host cells and becomes visible as a necrotic lesion (Curtis \& Wolpert, 2004; van Baarlen et al., 2004). In this situation, PCD shows specific apoptosis hallmarks, which include cell shrinkage and membrane blebbing with retention of plasma membrane integrity, loss of mitochondrial membrane potential and release of cytochrome c into the cytoplasm, protease activation, chromatin condensation and nucleosome cleavage, decrease in ATP, and increase in reactive oxygen species (ROS; Dickman et al., 2017). Necrotrophic pathogens, such as fungi of the genus Botrytis, exploit the plant PCD pathway by actively triggering apoptosis in host cells through the activity of an array of effector compounds, allowing the pathogen to acquire nutrients from the dead plant cells and to colonize the plant tissue (Veloso \& van Kan, 2018).

To determine the level of resistance or susceptibility of a given plant genotype, assessment of disease symptoms in an infection assay is a key step to evaluate the outcome of an interaction between host and pathogen. Thus, visualization and quantification of PCD over a given period of time are essential to understand how the plant responds upon contact with the pathogen. Furthermore, a visual assessment is often performed to study the effect of individual pathogen IPs (including effectors) on the host, which is done by applying such molecules to a plant tissue by either agroinfiltration or IP injection. This approach allows plant genotypes to be compared for their responses to pathogens or specific IPs and for disease development or the establishment of resistance responses to be monitored over time.

At present, the most widely used and commonly accepted method to visualize plant cell death is the trypan blue staining (TBS) technique, which is a cell viability test based on the principle that live cells, possessing intact and functional cell membranes, exclude trypan blue dye whereas dead cells do not. This staining method was first described by Ehrlich (1885) in a study in which trypan blue dye was injected into animal tissue and he observed that the brain tissue showed less staining. Subsequent research showed that when trypan blue was injected directly into the central nervous system instead, the brain tissue would stain equally well, but the stain would not travel to the rest of the body, showing a compartmentalization between the cerebrospinal fluid and the vasculature in the rest of the body (Ehrlich, 1885; Mott, 1913). In the course of the 20th century and until current times, TBS has been successfully implemented in plant biology and phytopathological studies because it was found that TBS allows plant vascular tissue (Escamez et al., 2017), dead Arabidopsis thaliana cells as a consequence of HR induced by Pseudomonas syringae effectors (Johansson et al., 2015), or cell death responses in Brassica rapa cotyledons to oomycete elicitins (Takemoto et al., 2005) to be visualized. TBS consequently became useful to assess the extent of plant tissue colonization by microbial pathogens and, for example, for detecting the presence of (micro-)lesions in plant-microbe interactions (Vogel et al., 2005). Nevertheless, TBS has four major drawbacks. First, it is not understood how trypan blue dye can pass membranes of dead cells as well as of hyphae of live fungi and oomycetes, whereas the dye is specifically excluded from live plant cells with an intact plasma membrane. 
Second, the method requires the use of toxic chemicals, such as the dye itself, phenol, and chloral hydrate. Third, TBS represents a destructive assay that does not allow disease progression and/or the occurrence of plant cell death responses to be followed in the same sample over time, nor does it allow the tissue to be sampled for RNA, protein, or metabolite analyses after it is stained. Finally, the method provides a qualitative test of cell viability, but it does not easily allow PCD to be quantified in a standardized way.

We here report a nondestructive, nontoxic, and reproducible method to visualize and quantify cell death in green plant tissues. This method can be implemented to study different pathosystems and allows the development of PCD to be followed over time. The protocol is based on the detection of light signals emitted by plant cells undergoing cell death. With the aid of a fluorescence imaging system, we observed red light emission by leaf tissue upon infiltration with known PCD-inducing compounds, agroinfiltration, or infection by a pathogen. We provide evidence that the red light signal originates from the increase in chlorophyll fluorescence due to dismantling of chloroplast thylakoid membranes in cells undergoing PCD. We provide several illustrations that this method can be used as a versatile tool for quantitative and nondestructive evaluation of plant responses to microbial pathogens or their IPs.

\section{RESULTS}

This study was initiated after the observation that on exposure to a green light source, leaf tissue undergoing a typical HR emits light that could be detected using the red light filters of a fluorescence imaging system. Our initial experiments indicated that the intensity of the emitted light positively correlated with the severity of visual signs of cell death. We set out to explore whether this method would be suitable for the development of an alternative, fast, simple, and safe nondestructive tool to visualize cell death in plant tissue. This tool should allow users to quantify PCD in a standardized and reproducible manner and to follow the development of PCD over a given time course. Therefore, we first tested whether the areas yielding a light signal that was detected with red light imaging originated from the affected tissue that in PCD tests is normally stained with trypan blue. Nicotiana benthamiana:Cf-4 leaves were infiltrated with pure Avr4 protein from C. fulvum and we compared the visible HR symptoms with the classic TBS and the red light signal on the same leaves (Figure 1). At 3 days after infiltration (dai), HR symptoms were observed on leaves infiltrated with $5 \mu \mathrm{M}$ of Avr4 but not $1 \mu \mathrm{M}$ (Figure 1, left column). Comparing TBS (Figure 1, middle column) to the red light signal (Figure 1, right column), it can be noticed that the areas stained with trypan blue exactly match with the areas from which the red light signal is detected by the imaging system. The signal intensity was clearly stronger at higher Avr4 concentration and showed a punctate pattern, with the most intense red light emission from areas that displayed the most obvious signs of cell death.

Recently, several studies have used UV transillumination to monitor cell death responses (Guo et al., 2020; Zdrzalek et al., 2020). We compared the use of UV light as a source of excitation for imaging of tissue undergoing PCD to the imaging based on green light excitation described above (Figure S1). When the leaves were excited with UV light, a much stronger background signal was detected in the healthy tissue and in the leaf veins. Such a background signal interferes with the red light signal that derives from tissue undergoing PCD, thereby rendering the UV imaging system less sensitive for areas yielding lower signal intensities. (a)

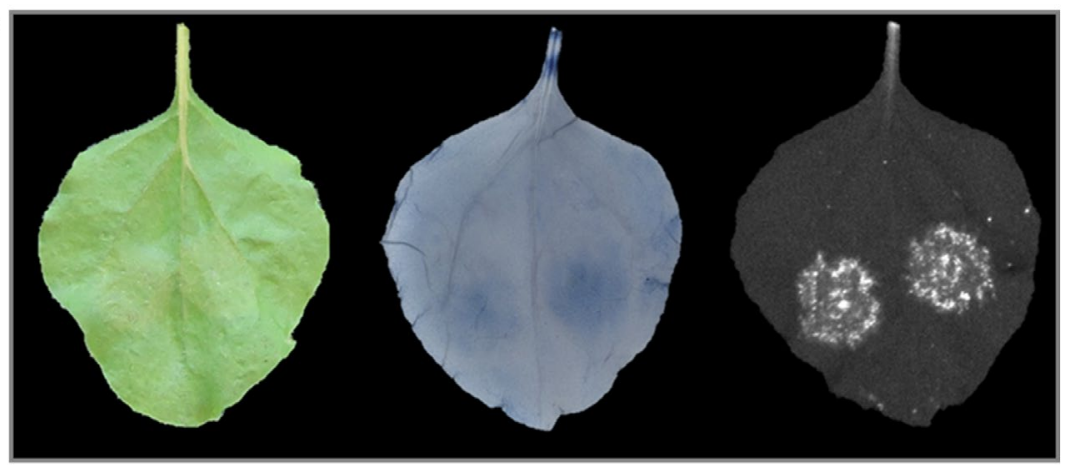

FIGURE 1 Cell death symptoms caused by Avr4 infiltration (a: $1 \mu \mathrm{M}$; b: $5 \mu \mathrm{M}$ ) in leaves of Nicotiana benthamiana:Cf-4 plants, visualized at 3 days after infiltration with visible light (left column), trypan blue staining (middle column), and red light imaging system (right column)

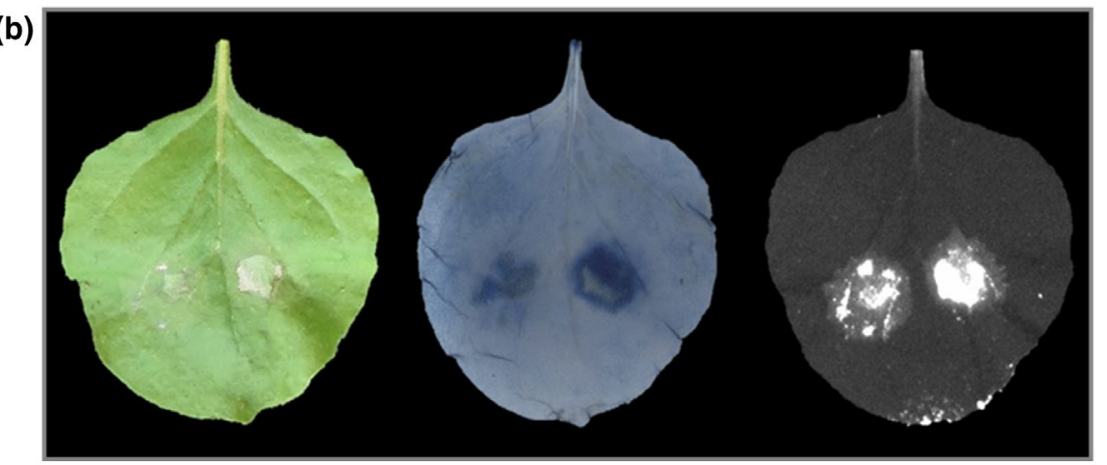


Having shown that the necrotic areas exactly match with the areas from which the red light signal originates, we infiltrated different IPs into leaves of $N$. benthamiana to test whether the intensity of the red light signal correlates with the severity of the visible PCD symptoms (Figures 2-4). On infiltration of flg22, at 3 dai no cell death symptoms were observed on the leaves, and also no red light signal was detected (Figure 2a). On the other hand, a clear red light signal was detected on Avr4 infiltration in leaves of $N$. benthamiana stably expressing $\mathrm{Cf}-4$, but, apart from some chlorosis, no cell death symptoms were observed (Figure $2 \mathrm{~b}$ ). In contrast, BcNEP1 infiltration caused the swift development of a clearly visible area of dry necrotic tissue that matched with the area yielding a strong red light signal (Figure 2c). In a ROS burst assay, the incubation of leaf disks of $N$. benthamiana in a solution containing either flg22, Avr4, or BcNEP1 triggered a strong ROS burst (Figure 2, lower panel).

To test whether this method can also be used in combination with Agrobacterium transient transformation assays (ATTA; agroinfiltration), genes encoding IPs and their corresponding immune receptors were transiently expressed in $\mathrm{N}$. benthamiana leaves and the development of HR was monitored. Expression of Avr4 or Cf-4 alone caused no symptoms of HR and no red light signal was detected outside the area of wounding caused by the syringe during the infiltration. Transient expression of both Avr4 and Cf-4 in the same leaf area caused symptoms of $\mathrm{HR}$ and a clear red light signal was detected (Figure 3a). Similarly, transient expression of the protein Avr4BS4 from Xanthomonas campestris pv. vesicatoria and its matching tomato immune receptor, the NB-LRR BS4, triggered symptoms of HR and a red light signal only when infiltrated in combination (Figure $3 \mathrm{~b}$ ) (Schornack et al., 2004). Moreover, the elicitor-independent HR triggered by $\mathrm{NRC}^{\mathrm{D} 481 \mathrm{~V}}$ was also tested, which is a constitutively active mutant of the tomato NB-LRR NRC1. In the agroinfiltrated areas, HR symptoms were observed together with a strong emission of a red light signal. In contrast, expression of $\mathrm{NRC}^{\mathrm{K} 191 \mathrm{R}}$, which is an inactive mutant of NRC1, caused no HR and no red light signal was detected (Figure 3c) (Gabriëls et al., 2007). Furthermore, quantification of HR upon agroinfiltration was assessed by transiently expressing Avr4 in N. benthamiana:Cf-4 after virus-induced silencing of the gene encoding the immune receptor Cf-4. A significant decrease in the signal intensity was observed when Cf-4 was silenced (Figure $3 d$ ).

To explore whether the imaging system also functions for plants other than N. benthamiana, we infiltrated a different IP, namely the Botrytis cinerea polygalacturonase 3 (BcPG3) protein, in detached leaves of wild-type (WT) Arabidopsis Col-0 plants and in an Atsobir1 Arabidopsis mutant (Zhang et al., 2014). As depicted in Figure S2, necrotic tissue in combination with a clear red light signal was observed only in leaves of the WT plants, whereas neither necrotic spots nor a red light signal were detected on BcPG3 infiltration in the Atsobir1 mutant. In addition, a specific red light signal was detected in leaves of transgenic tomato seedlings systemically expressing both the C. fulvum Avr4 and the Cf- 4 resistance protein (Joosten et al., 1994), even before symptoms of necrosis were observed (Figure S3).

Besides the qualitative visualization of PCD in different plant species, this methodology should also allow the quantification of the magnitude of PCD by measuring the intensity of the red light signal. To test this application, we infiltrated leaves of N. benthamiana:Cf-4 with different concentrations of Avr4 protein from C. fulvum and BcNEP1 protein from B. cinerea (Figure 4). While even the highest concentration $(1 \mu \mathrm{M})$ of Avr4 protein barely induced any sign of PCD as observed with the naked eye (Figure $4 d$ ), the imaging system detected increasing red

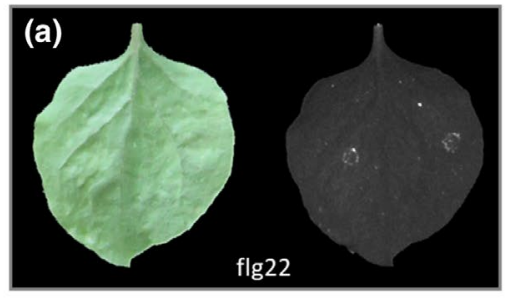

(d)

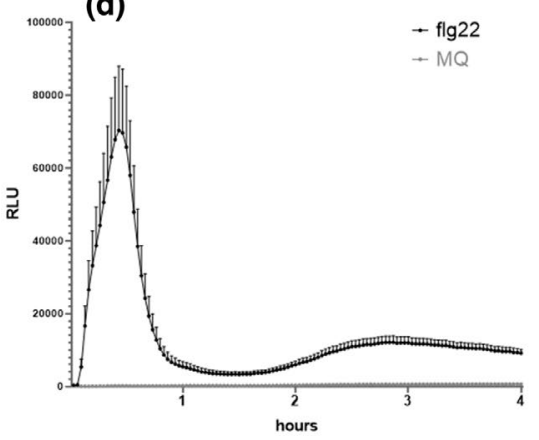

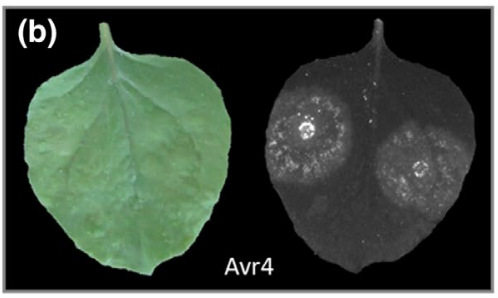

(e)

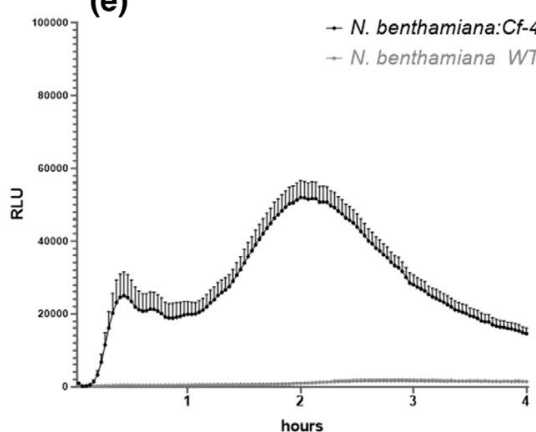

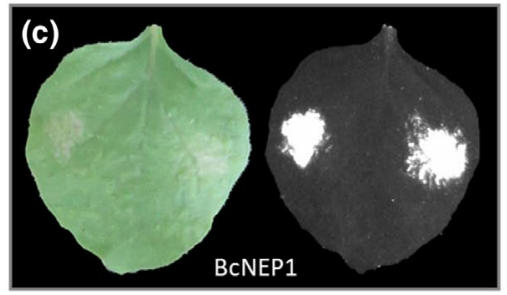

(f)

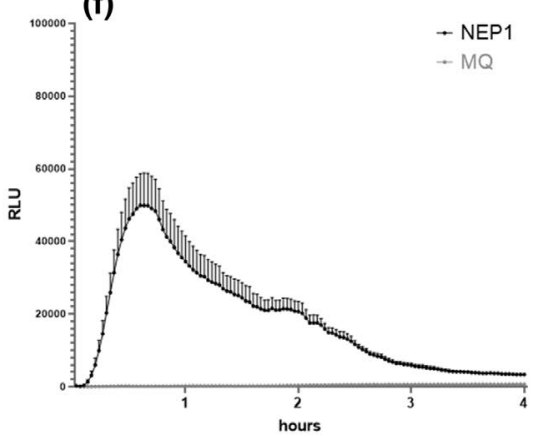

FIGURE 2 Visible cell death symptoms (left) and detected red light signal (right) on infiltration of $0.1 \mu \mathrm{M}$ flg22 (a), $0.01 \mu \mathrm{M}$ Avr4 (b), and $0.004 \mu \mathrm{M}$ BcNEP1 (c) at 3 days after ifiltration in leaves of Nicotiana benthamiana:Cf-4. Reactive oxygen species burst triggered by $0.1 \mu \mathrm{M}$ flg22 and the Milli-Q water (MQ) control in N. benthamiana wild-type (WT) (d), $0.01 \mu$ M Avr4 in N. benthamiana:Cf-4 and the N. benthamiana WT control plant (e), and $0.004 \mu \mathrm{M} \mathrm{BcNEP1}$ and the MQ control in N. benthamiana WT (f). $n=8$, error bars show the SE 
(a)

Avr4

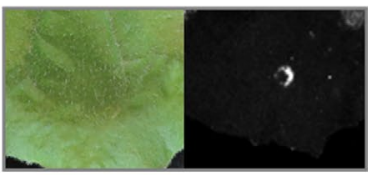

(b)

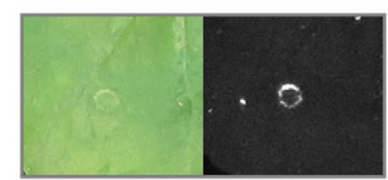

(c)

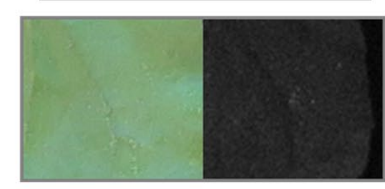

Cf-4

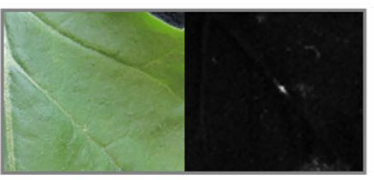

AvrBs4



$N R C 1^{\text {D481V }}$

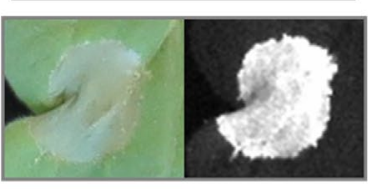

(d)

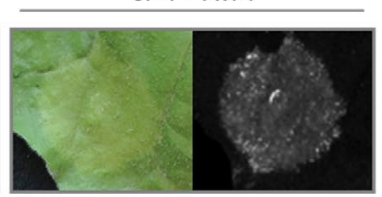

Bs4 + AvrBs4

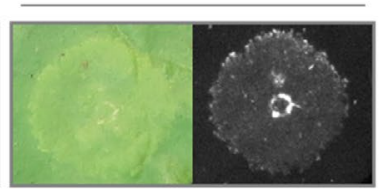

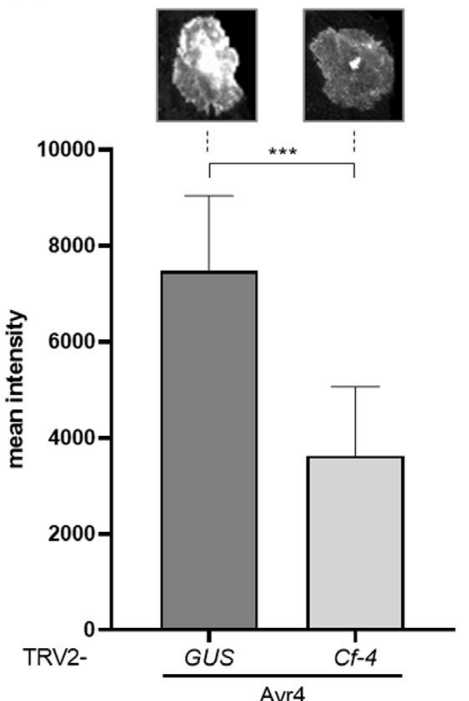

FIGURE 3 Cell death symptoms on agroinfiltration of Avr4/Cf-4 (a), AvrBS4/BS4 (b), NRC1 ${ }^{\text {K191R }}$ and NRC1 ${ }^{\text {D481V }}$ (c) in Nicotiana benthamiana leaves at 3 days after infiltration (dai), visualized under visible light and with the red light imaging system. (d) Red light signal intensity measured upon agroinfiltration of Avr 4 after virus-induced gene silencing of $C f-4$ in N. benthamiana:Cf- 4 at 3 dai, $n=8$. Bars represent SD. ${ }^{* *}$ Significant difference using a t test, $p<.001$
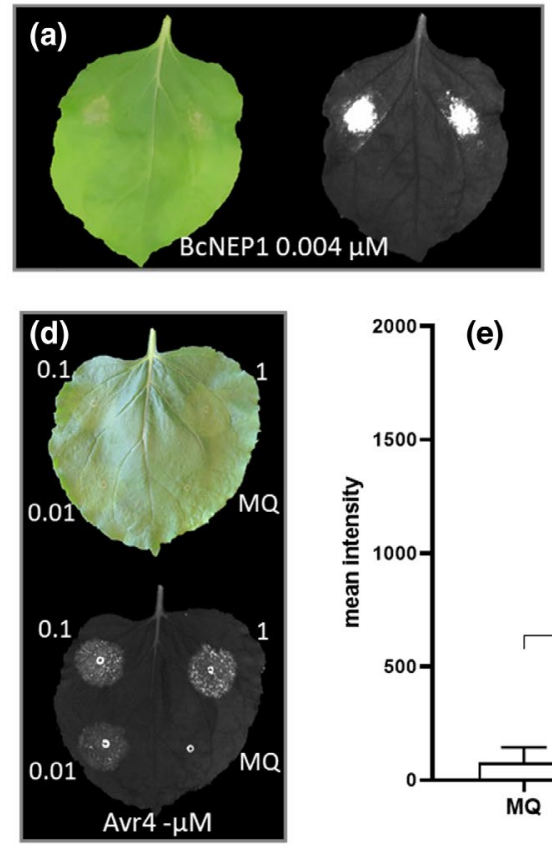
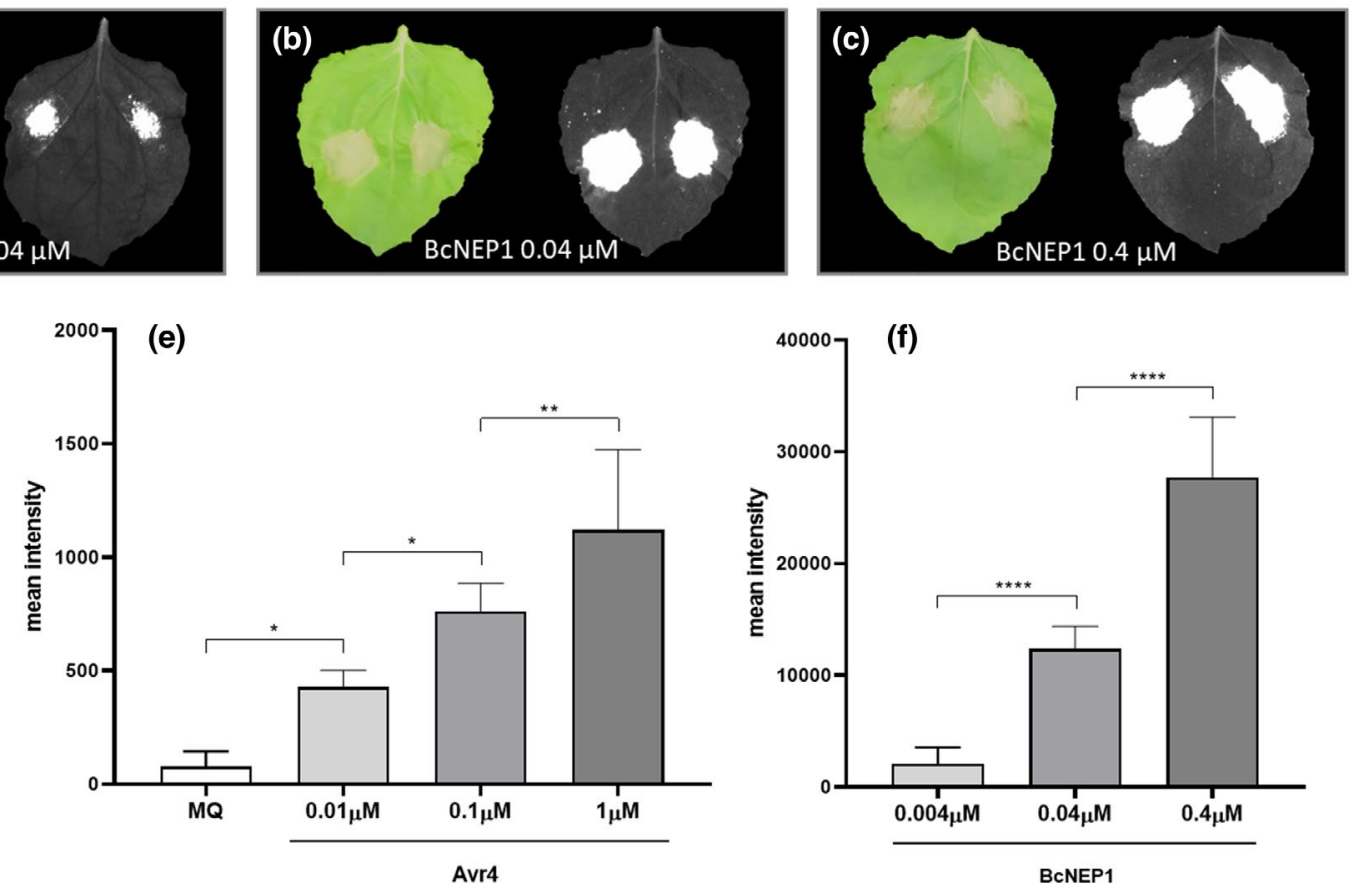

FIGURE 4 Dose-dependent cell death symptoms caused by BcNEP1 (a, b, c) and Avr4 (d) protein infiltration in Nicotiana benthamiana:Cf-4 at 3 days after infiltration, as visualized under visible light and with the red light imaging system. Quantification of red light signal intensities emitted by N. benthamiana:Cf-4 leaves infiltrated with 0.01, 0.1, and $1 \mu \mathrm{M}$ Avr4 protein and Milli-Q water (MQ) as a control (e), and with $0.004,0.04$, and $0.4 \mu \mathrm{M}$ BcNEP1 protein (f). Bars represent averages $\pm S D(n=8)$. Significant difference using analysis of variance followed by Tukey's test, ${ }^{*} p<.05,{ }^{* *} p<.01,{ }^{* * * *} p<.0001$

light signal intensities emitted by the infiltrated tissue on infiltration of higher Avr4 concentrations (Figure 4d,e). By contrast, no red light signal was detected in the mock-infiltrated areas, except at sites where the syringe was applied and pressed on the leaf to infiltrate the Milli-Q water (MQ), thereby causing some wounding (Figure $4 d, M Q$ ). On the other hand, BcNEP1 infiltration caused visible necrotic spots even at the lowest concentrations used (Figure 4a), whereby the red light signal intensity measured on BcNEP1 infiltration was up to 25-fold higher than in Avr4 infiltrated leaves (Figure 4e,f). In addition, it can be noticed that when BcNEP1 was infiltrated at higher concentrations no clear 
differences in the necrotic tissues could be observed (Figure 4b,c). However, when quantified with the imaging system, increasing red light signal intensities were recorded in leaves infiltrated with increasing BcNEP1 concentrations (Figure 4f).

Furthermore, to monitor the development of PCD over a given time course of infection, leaves of $\mathrm{N}$. benthamiana and Arabidopsis Col-0 were inoculated with a conidial suspension of B. cinerea. In both cases, development of necrotic lesions was observed from 2 days postinoculation (dpi) and the imaging system detected a clear red light signal at the site of the lesion (Figure 5a,b). For N. benthamiana, pictures of the same detached leaves were taken at 2, 3, and $4 \mathrm{dpi}$. This was not possible for Arabidopsis leaves because they were too fragile after detachment, and therefore symptom development was followed on detaching different leaves at 2 and $3 \mathrm{dpi}$. Following incubation, we observed that the lesions expanded and that the red light signal was sometimes detected in irregular shapes, outside of the circular necrotic spot caused by the fungus. The latter light-emitting zones were not visible to the naked eye (Figure 5a,b, yellow arrows). Moreover, the imaging system detected a red light signal for both Arabidopsis and N. benthamiana, which was visible as concentric rings of different intensities at the location where the necrotic lesions developed (Figure 5a,b, red arrows). In addition to leaves, the red light imaging system was also tested to detect disease symptoms on stem tissue. When B. cinerea conidia were inoculated onto N. benthamiana intact and cut stems, red light emission was observed in stem tissue showing visible disease symptoms (Figure S4).

We also followed the development of necrotic lesions on two lily cultivars inoculated with two different Botrytis elliptica isolates.
Figure 6 shows the necrotic lesions that were observed at 3 and $4 \mathrm{dpi}$. In all cases the lesions expanded during the incubation. With both isolates, distinct areas were observed emitting a light signal of different intensities, with a fairly abrupt transition between them (Figure 6b). The outer area showed a lower red light intensity (Figure $6 \mathrm{~b}$ ) and this was not visible as necrotic tissue when the picture was taken with visible light. Moreover, for the most aggressive isolate (Be9401) in both cultivars the imaging system detected a much weaker red light signal in the centre of the lesion compared to the concentric rings around the inoculation site and the red light signal was also detected in leaf veins. In contrast, for the less aggressive isolate (Be9732), the signal remained intense at the inoculation site, while the lesions were still expanding and no red light signal was detected in the leaf veins. To illustrate the differences in symptom severity and to compare isolate virulence and cultivar susceptibility, the signal intensities over the lesion areas were quantified and the values were plotted on a bar chart (Figure S5).

To explain the origin and nature of the observed red light signal in tissue undergoing PCD, we hypothesized that the signal might derive from the disassembly of thylakoid membranes of the chloroplasts. To test this hypothesis, chloroplasts were isolated from $N$. benthamiana leaves and treated with different concentrations of the membrane-disrupting surfactants Nonidet P-40 and sodium dodecyl sulphate (SDS), and the surfactant solutions were also directly infiltrated in detached $N$. benthamiana leaves (Figures S6 and S7). As shown in Figure 7a,c, red light emission was detected in the chloroplast suspension after treatment with both surfactants, whereas no red light was emitted by the untreated (a)

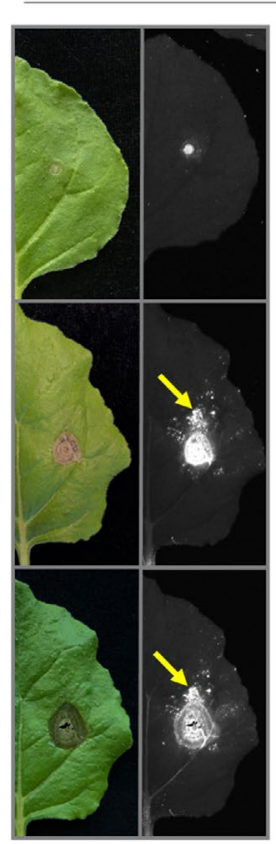

B. cinerea

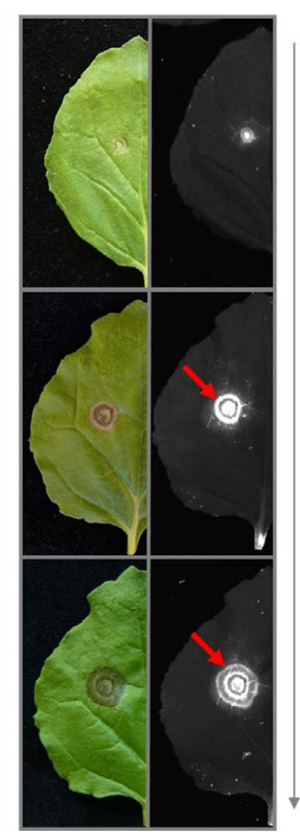

(b)

\section{B. cinerea}

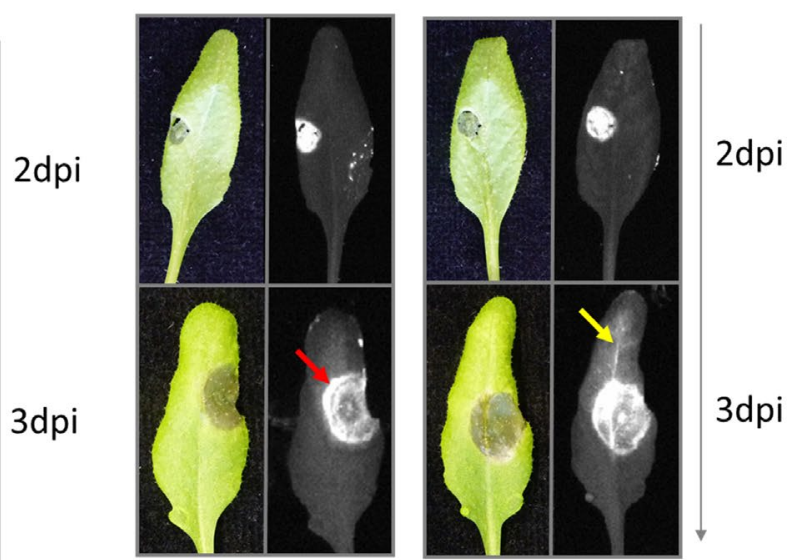

FIGURE 5 Necrotic lesions developing after Botrytis cinerea inoculation on leaves of (a) Nicotiana benthamiana and (b) Arabidopsis, visualized with visible light (left) and with the red light imaging system (right). Yellow arrows indicate the red light signal detected in tissue outside the necrotic lesions; red arrows indicate distinct concentric rings with different red light signal intensities within the lesion. dpi, days postinoculation 
(a)

Be9401

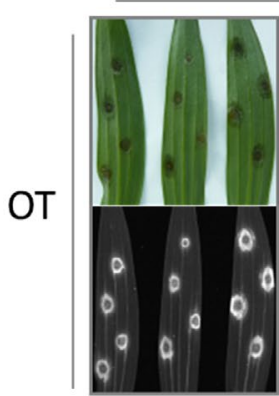

LO

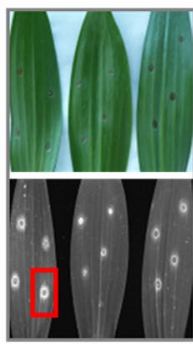

3dpi
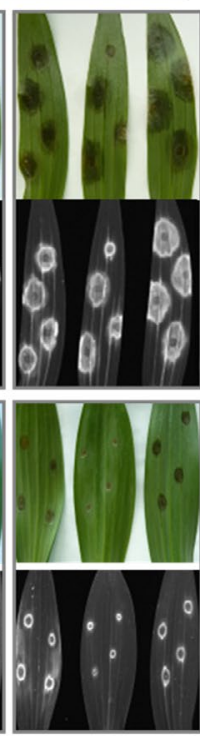

$4 d p i$
Be9732
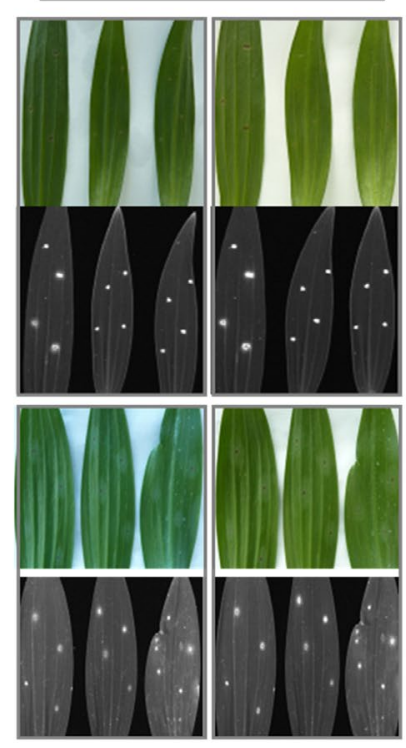

3dpi (b)

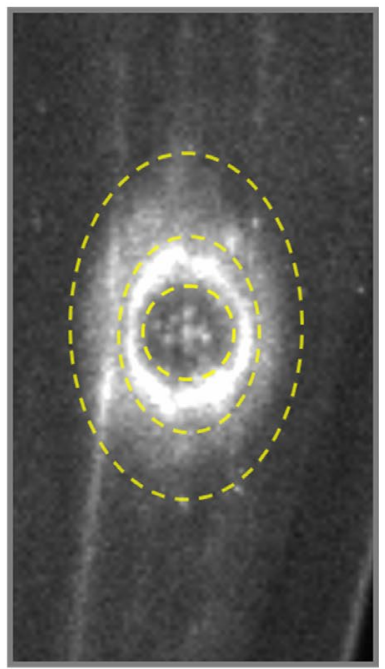

FIGURE 6 (a) Necrotic lesions developed after inoculation of Botrytis elliptica isolates Be9401 and Be9732 on leaves of lily genotypes OT and LO, visualized with visible light (upper) and with the red light imaging system (bottom), at 3 and 4 days postinoculation (dpi). For each time point and cultivar, three representative leaves are shown. (b) Magnification of a necrotic lesion observed at 3 dpi on genotype LO, highlighted by a red rectangle in (a), showing an area with different light signal intensities. Yellow dashed lines indicate the borders between these areas

chloroplast suspension and the pure surfactant solutions. The graphs in Figure 7b,d show that the red light intensities increased when the chloroplasts were treated with a higher concentration of the surfactant, as well as with a longer incubation time with $10 \%$ Nonidet P-40, and with $10 \%$ and $20 \%$ SDS. On direct leaf infiltration with the surfactant solutions, the tissue collapsed in a dose-dependent manner and eventually showed a brownish colouration that resembled the necrotic tissue observed upon infiltration of various IPs and in the inoculation assays (Figures S3-S5).

\section{3 | DISCUSSION}

\section{1 | Advantages and applications of red light imaging for studies in plant pathology}

Various imaging systems have been developed to investigate plant defence responses. These include quantification methods for ion flux changes and ROS accumulation (Grant \& Loake, 2000), nitric oxide generation (Delledonne et al., 1998), and biophoton generation (Bennett et al., 2005). Despite focusing on plant defence responses, none of these methods allows PCD to be investigated as a determinant of the outcome of the interaction in a given pathosystem, which usually requires a destructive staining method, TBS. In this study we describe a simple methodology to visualize and quantify PCD in green plant tissues using the red light filter of a fluorescence-luminescence imaging system, which was originally purchased for imaging DNA or protein gels, and western blots. We show that the system can also be applied for imaging plant cell death during interactions with pathogens or their IPs.

Compared to classical TBS, the red light imaging method has several advantages. First of all, it is safer because it does not require the use of toxic chemicals for preparing the staining and destaining solutions, and it generates no chemical waste. The method is fast and simple because it is solely based on the detection of a light signal that is automatically processed by the imaging system. This allows the analysis of a large number of samples in a short time, which makes it useful for large-scale screenings, with up to hundreds of leaves per day. Importantly, in contrast to TBS, this method is nondestructive and the same samples can be analysed at different time points to monitor the progression of PCD or the imaging may assist in selecting different regions in the sample for extraction of RNA, proteins, or metabolites. Both in the case of leaf infiltration with different IPs and in inoculation assays with pathogens, the method allows the plant response to be observed at different time points, thereby enabling the development of symptoms to be followed during the interaction over a given time span (Figures 5 and 6). The red light imaging system allows PCD to be visualized and quantified in a standardized manner. Different parameters, such as the exposure time and the focus on particular sites of the sample, can be set in advance and with the aid of software the red light intensities can be quantified for comparison among different samples. On the other hand, for lesion size measurements, it is possible to quantify the area of the tissue for which the red light signal exceeds background levels. In contrast, in TBS the staining intensity may depend on how long the tissue is treated with the staining and destaining solutions, 
(a)
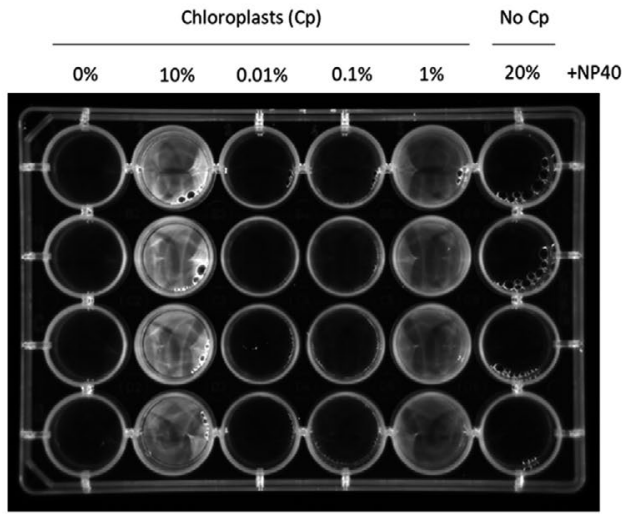

(b)

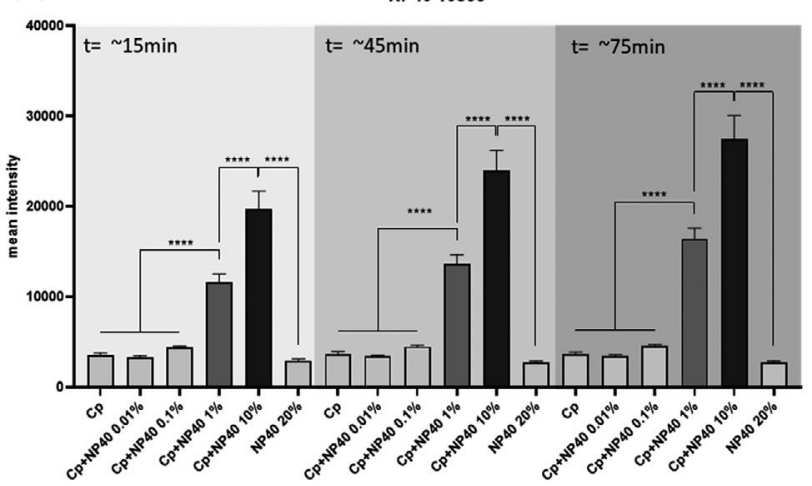

(c)



(d)

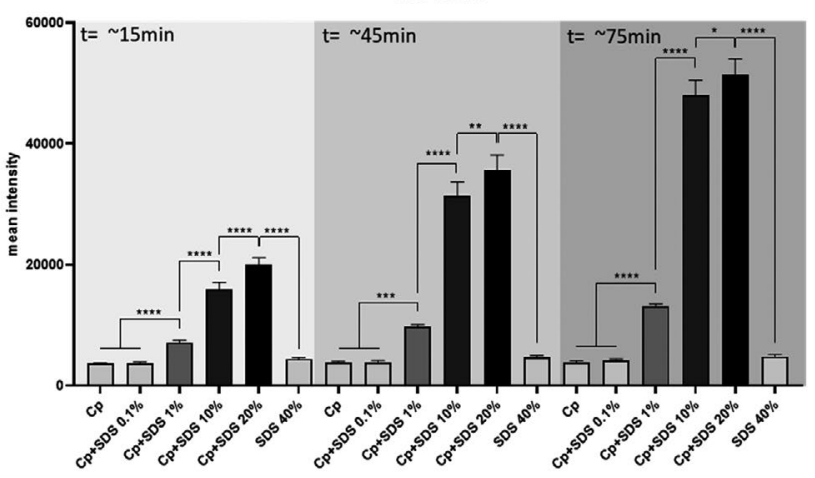

FIGURE 7 Red light emission in a 24-well plate containing a chloroplast suspension obtained from Nicotiana benthamiana leaves after treatment with Nonidet P-40 (NP40) (a, b) or sodium dodecyl sulphate (SDS) (c, d) at different concentrations. Red light signal intensities, determined at three time points after the indicated treatments, were quantified and bars represent averages $\pm S D(n=4)$. Significant difference using analysis of variance followed by Tukey's test, ${ }^{*} p<.05,{ }^{* * *} p<.001,{ }^{* * * *} p<.0001$

thereby only permitting a qualitative visualization of PCD, but hampering a quantitative comparison across multiple samples. It is important to note that the imaging system requires placing the samples on a flat surface, therefore it is not possible to acquire images from three-dimensional objects such as whole plants because in that case the device is unable to bring the complete area to be studied into focus. More specialized and expensive equipment would be needed for such purposes.

This simple and nondestructive method allows new research aimed at studying the role of PCD in plant-pathogen interactions to be pursued. PCD symptoms can be detected before becoming visible to the naked eye and different stages of PCD might be able to be distinguished depending on light intensities. In necrotic lesions caused by Botrytis spp., distinct concentric areas were observed showing a red light signal of different intensity. The absence of a red light signal at the centre of the lesion (Figure $6 \mathrm{~b}$ ) indicates that at that location the light signal emitting compounds have been completely degraded. In some cases, when moving distally from the centre of the lesion, the first area surrounding the inoculation site showed a higher signal intensity as compared to the lesion periphery, resulting in the formation of a concentric ring (Figure 6b). This observation might indicate that PCD is more advanced in the brighter region and is only just induced in the peripheral area. It remains to be established whether higher signal intensities correlate with more advanced stages of PCD. Moreover, by inoculating plant tissue with transgenic pathogen strains expressing green fluorescent protein (GFP), it should be possible to investigate whether host tissue colonization has already occurred in the areas displaying low-intensity red light signals, or whether PCD has been induced by effector compounds that are secreted by the pathogen and have moved into the surrounding host tissue.

We tested the versatility of the methodology for investigating PCD development by infiltration of different IPs. Upon flg22 infiltration, we observed neither a red light signal nor development of a necrotic lesion, despite the fact that flg22 recognition took place in the leaf tissue and triggered a ROS burst as previously reported (Figure 2) (Gómez-Gómez et al., 1999). This observation confirms that PCD activation requires specific signalling mechanisms to be induced and that not all IPs equally trigger PCD (van der Burgh \& Joosten, 2019). There was a dose- and time-dependent cell death response on infiltration of various IPs at different concentrations (Figures 1 and 4). Because the symptoms are not always clearly visible, the red light filter of the imaging system provides a more reliable and objective tool to quantitatively determine the contribution of given IPs to PCD induction in the host tissue. Finally, this method will help to investigate the function of pathogen-secreted effectors. For example, by labelling pathogen-secreted effectors with fluorescent tags, it will be possible to study their mobility in the tissue and 
localization over time during host infection, in conjunction with the host response to these effectors. Additionally, by identifying areas that are in an early stage of PCD, showing low-intensity red light signals, it should be possible to specifically dissect and process tissue areas to isolate proteins or RNAs at the appropriate time and location. Possibly, in this way also specific effector targets in the host cells and PCD signalling components can be identified via coimmunoprecipitation assays, as the appropriate tissue can be collected using the imaging system.

\section{2 | PCD, chlorophyll fluorescence, and the red light signal}

This study shows that tissue undergoing PCD emits red light that can be detected by means of the imaging system. We hypothesized that the red light signal derives from increased chlorophyll fluorescence, as a consequence of the disassembly of the thylakoid membranes in the chloroplasts. This hypothesis was tested by treating isolated chloroplasts with membrane-disrupting surfactants (Figure 7) and by leaf infiltration with such surfactants (Figures S6 and S7). The membrane-disrupting properties of the surfactants caused a concentration-dependent emission of the red light signal from isolated chloroplasts and infiltrated leaves. From a photochemical point of view, this phenomenon can be explained by the abortion of photochemical activity due to the dismantling of the chloroplast thylakoid membranes in cells undergoing PCD, resulting in an increase in chlorophyll fluorescence. In photochemically active plant cells, chlorophyll molecules are tightly packed together in light harvesting complexes (LHCs) that are located at the thylakoid membranes of the chloroplasts. Chlorophyll fluorescence is quenched by photochemical activity when LHC-photosystem II (PSII) complexes are assembled in thylakoids. Chlorophylls have two distinct absorption wavelength peaks in the visible light spectrum: the highest is found in the blue spectrum at c. $450 \mathrm{~nm}$ and the lowest at $650 \mathrm{~nm}$ in the red spectrum. Moreover, chlorophylls possess a fluorescence emission peak in the red spectrum at c.680 nm. During PCD or on perturbation of thylakoid membrane stability, LHC-PSII supercomplexes are disassembled from chloroplast thylakoid membranes. This causes cessation of the electron flow required for photochemical activity because the connection between PSII and photosystem I via plastoquinone, cytochrome-b6f, and plastocyanin is lost. As consequence, light-excited chlorophyll molecules will dissipate photon energy through heat and fluorescence (Baker, 2008).

\section{3 | Conclusion}

In this study we show that red light fluorescence detection by a multipurpose imaging system can be used to visualize plant tissue undergoing cell death. Quantifying the intensity of the red light signal enables the magnitude of cell death to be evaluated and thereby provides a readout of the plant immune response in a faster, safer, and nondestructive manner when compared to previously developed chemical staining methodologies. This application can be implemented to screen for differences in symptom severity in plantpathogen interactions, and to visualize and quantify in a more sensitive and objective manner the intensity of the plant response on perception of a given immunogenic pattern.

\section{4 | EXPERIMENTAL PROCEDURES}

\section{1 | Plant material and growth conditions}

Transgenic N. benthamiana plants stably expressing Cf-4 (N. benthamiana: Cf-4) (Gabriëls et al., 2006) and WT N. benthamiana plants were grown under $16 \mathrm{hr}$ light at $24^{\circ} \mathrm{C}$ and $8 \mathrm{hr}$ darkness at $22^{\circ} \mathrm{C}$ and $\mathrm{RH}$ of $75 \%$.

Arabidopsis ecotype Col-0 and the Atsobir1 mutant line in the Col-0 background (Gao et al., 2009), were grown under $12 \mathrm{hr}$ light at $21^{\circ} \mathrm{C}$ and $12 \mathrm{hr}$ darkness at $19^{\circ} \mathrm{C}$ at $\mathrm{RH}$ of $75 \%$.

Lilium genotypes from the hybrid types OT (Oriental $\times$ Trumpet) and LO (Longiflorum $\times$ Oriental) were planted as bulbs and grown in the greenhouse under natural day/night light regime and temperatures. Mature leaves of 2-month-old plants were used for the inoculation assays.

\section{2 | Red light imaging and signal quantification}

Treated plant material was placed in a ChemiDoc MP imaging system, model Universal Hood III (Bio-Rad), and images were acquired on excitation by a light source in the green visible spectrum (Green LED Module kit no. 1708284) or in the UV spectrum (UV-B Transillumination no. 1001361), with filters capturing the light emitted in the red visible spectrum (filter 605/50). The exposure time was adjusted to avoid image saturation. For comparison purposes, the images shown were autoscaled according to the treatment that yielded the lowest signal intensity.

For the IP infiltration assays, the mean signal intensity was calculated using Image Lab v. 6.0.1 software by manually selecting the treated areas and subtracting the background signal intensity value. For the disease assay in Lilium, the spots yielding a light signal were manually highlighted and the signal was calculated as the intensity over the selected area among 12 inoculations sites on three leaves per plant cultivar and per B. elliptica isolate at 2, 3, and $4 \mathrm{dpi}$.

\section{3 | Statistical analysis}

GraphPad Prism v. 8.4.0 was used to perform an analysis of variance (ANOVA) with a post hoc Tukey's test on the IP infiltration experiments, chloroplast treatment with surfactants, and the Botrytis disease assays in Lilium. 


\subsection{Immunogenic pattern-triggered cell death assays}

NEP1 protein from B. cinerea (BcNEP1) was produced in Pichia pastoris (Schouten et al., 2008) and dissolved in $10 \mathrm{mM}$ phosphate buffer, pH 5.5. The peptide flg22 (EZBiolab) and the protein Avr4 (produced in P. pastoris; van den Burg et al., 2001) from C. fulvum were dissolved in water. Protein infiltration was carried out in the first fully expanded leaf of 5- to 6-week-old N. benthamiana:Cf-4 and WT plants, and the cell death response was scored at 3 dai. The in filtrated areas were photographed and the average red light signal intensities were calculated.

B. cinerea polygalacturonase 3 (BcPG3) protein produced in P. pastoris (Zhang et al., 2014) was dissolved in $10 \mathrm{mM}$ sodium acetate buffer $\mathrm{pH} 4.2$ and infiltrated at a concentration of $1.5 \mu \mathrm{M}$ in fully expanded rosette leaves of 6- to 7-week-old Arabidopsis plants. The necrotic areas were photographed and analysed by red light imaging at 8 dai.

Tomato (Solanum lycopersicum) seedlings stably expressing both Avr4 and Cf- 4 were generated by crossing transgenic tomato cv. Moneymaker (MM) expressing C. fulvum effector Avr4 to MMCf-4 plants (Etalo et al., 2013; Stulemijer et al., 2007). After sowing, plants were grown for 4 weeks at $33^{\circ} \mathrm{C}$ and $100 \% \mathrm{RH}$ to suppress systemic PCD. Synchronized systemic PCD was eventually induced by transferring the seedlings to $20^{\circ} \mathrm{C}$ and $70 \% \mathrm{RH}$. The first fully developed tomato leaves were harvested $4 \mathrm{hr}$ after transfer and were photographed.

\section{5 | Agrobacterium-mediated transient transformation and virus-induced gene silencing in N. benthamiana}

Agrobacterium-mediated transient transformation (agroinfiltrations) were performed as previously described (van der Hoorn et al., 2000). The constructs pMOG800-Cf-4, pGWB20-BS4, pVsF-AvrBS4, pBINKS-NRC1 ${ }^{\mathrm{D} 481 \mathrm{~V}}$, and pBINKS-NRC1 $1^{\mathrm{K} 191 \mathrm{R}}$ GFP were infiltrated with Agrobacterium tumefaciens cultures at $\mathrm{OD}_{600}=1$. The construct pMOG800-Avr4 was infiltrated with A. tumefaciens cultures at $\mathrm{OD}_{600}=0.03$. Symptoms were evaluated at 3 dai.

Virus-induced gene silencing (VIGS) using tobacco rattle virus (TRV)-based vectors was performed in N. benthamiana:Cf-4 as previously described (Liebrand et al., 2012).

\section{6 | Trypan blue staining}

$N$. benthamiana leaves infiltrated with BCNEP1 protein were harvested at 3 dai and boiled for 2-5 min in staining solution, consisting of $16.6 \%$ (wt/vol) of trypan blue in an ethanol/lactic acid/phenol/ water (3:1:1:1) mixture. After this, the leaves were destained with chloral hydrate and photographed.

\section{7 | ROS burst measurements}

For each treatment, we used eight leaf discs $(5 \mathrm{~mm})$ punched from the first fully expanded leaf of 5- to 6-week-old N. benthamiana:Cf-4 plants. The discs were floated on $50 \mu$ of Milli-Q water (MQ) in a 96-well plate and kept in the dark at room temperature for $5 \mathrm{hr}$. The $\mathrm{MQ}$ was then removed using tissue paper, replaced by $50 \mu \mathrm{l}$ of $\mathrm{MQ}$, and kept in the dark for $1 \mathrm{hr}$ at room temperature. After this, $50 \mu \mathrm{l}$ of solution containing the IPs, $50 \mu \mathrm{M}$ luminol L-012 (FUJIFILM), and $10 \mu \mathrm{g}$ horseradish peroxidase were added to each well. The luminescence values were measured using a CLARIOstar plate reader (BMG LABTECH) over a period of $4 \mathrm{hr}$.

\section{8 | Disease assays with Botrytis spp.}

Leaves, intact and cut stems of $N$. benthamiana plants, and leaves of WT Arabidopsis Col-O were inoculated with conidia of $B$. cinerea B05.10. The fungus was grown on malt extract agar (50 g/L; Oxoid) and sporulation was induced by illumination with UV-A lamps. After harvesting, the concentration of the conidia was adjusted to $10^{6} / \mathrm{ml}$ using potato dextrose broth (PDB; Oxoid) at $24 \mathrm{~g} / \mathrm{L} \mathrm{PDB}$, and inoculated in 2- $\mu$ l droplets on the adaxial side of the leaves. Mock inoculation was carried out by applying $2-\mu$ ldroplets of PDB solution. Inoculated plants were then incubated in moist plastic boxes and were kept under the same light conditions under which the plants were grown before. Inoculated leaves were detached at $2 \mathrm{dpi}$, photographed, and analysed by red light imaging. The leaves were then stuck into wet floral foam and returned to the plastic boxes to allow disease progression to take place. This procedure was repeated at 3 and 4 dpi to visualize symptom development. Given the fragility of inoculated $A$. thaliana leaves, it was only possible to record symptom development for $N$. benthamiana leaves. Symptom development on inoculated $N$. benthamiana stems was evaluated at $4 \mathrm{dpi}$.

Detached leaves from two different cultivars of Lilium spp. (OT and LO) were abaxially inoculated with $2-\mu l$ droplets of B. elliptica conidia from isolates Be9401 and Be9732, respectively ( $10^{5}$ conidia/ $\mathrm{ml}$ in $12 \mathrm{~g} / \mathrm{L} \mathrm{PDB}$ ). Mock inoculation was carried out with $2-\mu \mathrm{l}$ droplets of PDB. Inoculated leaves were incubated in moist plastic boxes and pictures of red light imaging were taken at 3 and $4 \mathrm{dpi}$, with $2 \mathrm{~s}$ exposure. The signal intensities were analysed in a manually drawn area, encompassing the necrotic lesion for all the cultivar $\times$ pathogen combinations. An area of variable size was selected in each control leaf for subtraction of the background signal of healthy tissue.

\section{9 | Thylakoid disassembly test}

Intact chloroplasts were isolated from $\mathrm{N}$. benthamiana by stirring cut leaf pieces and following the protocol as described by $\mathrm{Ho}$ and Theg (2016). After ultracentrifugation, the Percoll layer containing the chloroplasts was transferred to a 24 -well plate with $150 \mu$ l aliquots 
per well. Two surfactants with membranolytic activity were added to each well to perturb thylakoid membrane stability. The surfactants were used at final concentrations of $0.01 \%, 0.1 \%, 1 \%$, and $10 \%$ for Nonidet P-40 (wt/vol $=\mathrm{g} / 100 \mathrm{ml}$ ) and $0.1 \%, 1 \%, 10 \%$, and $20 \%$ for SDS (wt/vol $=\mathrm{g} / 100 \mathrm{ml}$ ). One hundred and fifty microlitres of the respective surfactant at the different concentrations was added to the wells containing the chloroplast suspension. Untreated chloroplast suspension and pure surfactants at the highest concentration were used as controls. After gently mixing and 20 min of incubation in the dark, the microtitre plates were placed in the imaging system to detect the light signal using red light imaging, at an exposure time of $10 \mathrm{~s}$.

\section{ACKNOWLEDGEMENTS}

The research of S.L.V. is funded by the Peruvian Council for Science, Technology and Technological Innovation (CONCYTEC) and its executive unit FONDECYT, PhD scholarship contract 119-2017-FONDECYT. The research of M.C.M. is funded by Dutch NWO-Science domain (NWO-ENW), project GSGT.GSGT.2018.008.

\section{DATA AVAILABILITY STATEMENT}

The data that support the findings of this study are available from the corresponding author upon reasonable request.

\section{ORCID}

Matthieu H. A. J. Joosten (iD https://orcid. org/0000-0002-6243-4547

Jan A. L. van Kan iD https://orcid.org/0000-0003-3563-1550

\section{REFERENCES}

Baker, N.R. (2008) Chlorophyll fluorescence: a probe of photosynthesis in vivo. Annual Review of Plant Biology, 59, 89-113.

van Baarlen, P., Staats, M. \& van Kan, J.A.L. (2004) Induction of programmed cell death in lily by the fungal pathogen Botrytis elliptica. Molecular Plant Pathology, 5, 559-574.

Bennett, M., Metha, M. \& Grant, M. (2005) Biophoton imaging: a nondestructive method for assaying $\mathrm{R}$ gene responses. Molecular PlantMicrobe Interactions, 18, 95-102.

Boller, T. \& Felix, G. (2009) A renaissance of elicitors: perception of microbe-associated molecular patterns and danger signals by pattern-recognition receptors. Annual Review of Plant Biology, 60, 379-406.

van den Burg, H., de Wit, P.J.G.M. \& Vervoort, J. (2001) Efficient $13 \mathrm{C} / 15 \mathrm{~N}$ double labeling of the avirulence protein AVR4 in a methanol-utilizing strain (Mut+) of Pichia pastoris. Journal of Biomolecular NMR, 20, 251-261.

van der Burgh, A. \& Joosten, M.H.A.J. (2019) Plant immunity: thinking outside and inside the box. Trends in Plant Science, 24, 587-601.

Cai, Q., Qiao, L., Wang, M., He, B., Lin, F.M., Palmquist, J. et al. (2018) Plants send small RNAs in extracellular vesicles to fungal pathogen to silence virulence genes. Science, 360, 1126-1129.

Curtis, M. \& Wolpert, T. (2004) The victorin-induced mitochondrial permeability transition precedes cell shrinkage and biochemical markers of cell death, and shrinkage occurs without loss of membrane integrity. The Plant Journal, 38, 244-259.

Delledonne, M., Xia, Y., Dixon, R.A. \& Lamb, C. (1998) Nitric oxide functions as a signal in plant disease resistance. Nature, 394, 585-588.

Dickman, M., Williams, B., Li, Y., de Figueiredo, P. \& Wolpert, T. (2017) Reassessing apoptosis in plants. Nature Plants, 3, 773-779.
Ehrlich, P. (1885) Das Sauerstoff-Bedürfnis des Organismus. Eine farbenanalytische Studie. Berlin: August Hirschwald, 1885, 8 2 BI.

Escamez, S., Bollhöner, B. \& Tuominen, H. (2017) Quick histochemical staining methods to detect cell death in xylem elements of plant tissues. In: de Lucas, M. \& Etchhells, J. (Eds.) Xylem. Methods in Molecular Biology. New York: Humana Press, Vol 1544, pp. 27-36.

Etalo, D.W., Stulemeijer, I.J., van Esse, H.P., de Vos, R.C., Bouwmeester, H.J. \& Joosten, M.H.A.J. (2013) System-wide hypersensitive response-associated transcriptome and metabolome reprogramming in tomato. Plant Physiology, 162, 1599-1617.

Gabriëls, S.H., Takken, F.L., Vossen, J.H., de Jong, C.F., Liu, Q., Turk, S.C. et al. (2006) cDNA-AFLP combined with functional analysis reveals novel genes involved in the hypersensitive response. Molecular PlantMicrobe Interactions, 19, 567-576.

Gabriëls, S.H., Vossen, J.H., Ekengren, S.K., van Ooijen, G., Abd-ElHaliem, A.M., van den Berg, G.C.M. et al. (2007) An NB-LRR protein required for $\mathrm{HR}$ signalling mediated by both extra-and intracellular resistance proteins. The Plant Journal, 50, 14-28.

Gao M., Wang X., Wang D., Xu F., Ding X., Zhang Z. et al. (2009) Regulation of cell death and innate immunity by two receptor-like kinases in Arabidopsis. Cell Host \& Microbe, 6, 34-44.

Gómez-Gómez, L., Felix, G. \& Boller, T. (1999) A single locus determines sensitivity to bacterial flagellin in Arabidopsis thaliana. The Plant Journal, 18, 277-284.

Granado, J., Felix, G. \& Boller, T. (1995) Perception of fungal sterols in plants (subnanomolar concentrations of ergosterol elicit extracellular alkalinization in tomato cells). Plant Physiology, 107, 485-490.

Grant, J. \& Loake, G. (2000) Role of reactive oxygen intermediates and cognate redox signaling in disease resistance. Plant Physiology, 124, 21-30.

Grant, M., Brown, I., Adams, S., Knight, M., Ainslie, A. \& Mansfield, J. (2000) The RPM1 plant disease resistance gene facilitates a rapid and sustained increase in cytosolic calcium that is necessary for the oxidative burst and hypersensitive cell death. The Plant Journal, 23, 441-450.

Guo, Y., Dupont, P.Y., Mesarich, C.H., Yang, B., McDougal, R.L., Panda, P. et al. (2020) Functional analysis of RXLR effectors from the New Zealand kauri dieback pathogen Phytophthora agathidicida. Molecular Plant Pathology, 21, 1131-1148.

Gust, A., Pruitt, R. \& Nürnberger, T. (2017) Sensing danger, key to activating plant immunity. Trends in Plant Science, 22, 779-791.

Ho, J. \& Theg, S. (2016) The formation of stromules in vitro from chloroplasts isolated from Nicotiana benthamiana. PLoS One, 11, e0146489.

van der Hoorn, R.A., Laurent, F., Roth, R. \& de Wit, P.J.G.M. (2000) Agroinfiltration is a versatile tool that facilitates comparative analyses of Avr 9/Cf-9-induced and Avr 4/Cf-4-induced necrosis. Molecular Plant-Microbe Interactions, 13, 439-446.

Jain, D. \& Khurana, J. (2018) Role of pathogenesis-related (PR) proteins in plant defense mechanism. In: Singh, A. and Singh, I.K. (Eds.) Molecular aspects of plant-pathogen interaction. Singapore: Springer, pp. 265-281.

Jeandet, P. (2015) Phytoalexins: current progress and future prospects. Molecules, 20, 2770-2774.

Johansson, O., Nilsson, A., Gustavsson, M., Backhaus, T., Andersson, M. \& Ellerström, M. (2015) A quick and robust method for quantification of the hypersensitive response in plants. Peer J, 3, e1469.

Joosten, M.H.A.J., Cozijnsen, T.J. \& de Wit, P.J.G.M. (1994) Host resistance to a fungal tomato pathogen lost by a single base-pair change in an avirulence gene. Nature, 367, 384-386.

Jourdan, E., Henry, G., Duby, F., Dommes, J., Barthélemy, J.P., Thonart, P. et al. (2009) Insights into the defense-related events occurring in plant cells following perception of surfactin-type lipopeptide from Bacillus subtilis. Molecular Plant-Microbe Interactions, 22, 456-468.

Kabbage, M., Kessens, R., Bartholomay, L. \& Williams, B. (2017) The life and death of a plant cell. Annual Review of Plant Biology, 68, 375-404. 
Kanyuka, K. \& Rudd, J. (2019) Cell surface immune receptors: the guardians of the plant's extracellular spaces. Current Opinion in Plant Biology, 50, 1-8.

Liebrand, T.W., Smit, P., Abd-El-Haliem, A., de Jonge, R., Cordewener, J.H., America, A.H. et al. (2012) Endoplasmic reticulum-quality control chaperones facilitate the biogenesis of $\mathrm{Cf}$ receptor-like proteins involved in pathogen resistance of tomato. Plant Physiology, 159, 1819-1833.

Luna Diez, E., Pastor, V., Jérôme, R., Flors, V., Mauch-Mani, B. \& Ton, J. (2010) Callose deposition: a multifaceted plant defense response. Molecular Plant-Microbe Interactions, 24, 183-193.

Michaeli, S., Galili, G., Genschik, P., Fernie, A. \& Avin-Wittenberg, T. (2016) Autophagy in plants-what's new on the menu? Trends in Plant Science, 21, 134-144.

Mott, F.W. (1913) The late professor Edwin Goldmann's investigations on the central nervous system by vital staining. British Medical J, 2, 871-873.

Schornack, S., Ballvora, A., Gürlebeck, D., Peart, J., Ganal, M., Baker, B. et al. (2004) The tomato resistance protein Bs4 is a predicted non-nuclear TIR-NB-LRR protein that mediates defense responses to severely truncated derivatives of AvrBs4 and overexpressed AvrBs3. The Plant Journal, 37, 46-60.

Schouten, A., van Baarlen, P. \& van Kan, J.A.L. (2008) Phytotoxic NLPs from the necrotrophic fungus Botrytis cinerea associate with membranes and the nucleus of plant cells. New Phytologist, 177493-505.

Stulemeijer, I.J., Stratmann, J.W. \& Joosten, M.H. (2007) Tomato mitogen-activated protein kinases LeMPK1, LeMPK2, and LeMPK3 are activated during the $\mathrm{Cf}$-4/Avr4-induced hypersensitive response and have distinct phosphorylation specificities. Plant Physiology, 144, 1481-1494.

Takemoto, D., Hardham, A. \& Jones, D. (2005) Differences in cell death induction by Phytophthora elicitins are determined by signal components downstream of MAP kinase kinase in different species of Nicotiana and cultivars of Brassica rapa and Raphanus sativus. Plant Physiology, 138, 1491-1504.

Thomas, C., Jones, D., Parniske, M., Harrison, K., Balint-Kurti, P., Hatzixanthis, K. et al. (1997) Characterization of the tomato Cf-4 gene for resistance to Cladosporium fulvum identifies sequences that determine recognitional specificity in Cf-4 and Cf-9. The Plant Cell, 9, 2209-2224.

Turkan, I. (2018) ROS and RNS: key signalling molecules in plants. Journal of Experimental Botany, 69, 3313-3315.

Veloso, J. \& van Kan, J.A.L. (2018) Many shades of grey in Botrytis-host plant interactions. Trends in Plant Science, 23, 613-622.

Vogel, J., Raab, T., Somerville, C. \& Somerville, S. (2005) Mutations in PMR5 result in powdery mildew resistance and altered cell wall composition. The Plant Journal, 40, 968-978.

Voigt, C. (2014) Callose-mediated resistance to pathogenic intruders in plant defense-related papillae. Frontiers in Plant Science, 5, 168.

Zdrzalek, R., Kamoun, S., Terauchi, R., Saitoh, H. \& Banfield, M.J. (2020) The rice NLR pair Pikp-1/Pikp-2 initiates cell death through receptor cooperation rather than negative regulation. PLoS One, 15, e0238616.

Zhang, L., Kars, I., Essenstam, B., Liebrand, T.W., Wagemakers, L., Elberse, J. et al. (2014) Fungal endopolygalacturonases are recognized as microbe-associated molecular patterns by the Arabidopsis receptor-like protein RESPONSIVENESS TO BOTRYTIS POLYGALACTURONASES1. Plant Physiology, 164, 352-364.

\section{SUPPORTING INFORMATION}

Additional supporting information may be found online in the Supporting Information section.

How to cite this article: Landeo Villanueva S, Malvestiti MC, van leperen W, Joosten MHAJ, van Kan JAL. Red light imaging for programmed cell death visualization and quantification in plant-pathogen interactions. Mol Plant Pathol. 2021;22:361372. https://doi.org/10.1111/mpp.13027 